\title{
Does implementation of an early warning trigger system result in earlier administration of antibiotics for septic patients?
}

\author{
Julie Mackenhauer ${ }^{1 *}$, Anne-Katrine Bertelsen ${ }^{1}$, Helle Lykkeskov Nibro ${ }^{2}$, Troels Krarup Hansen ${ }^{1}$ \\ From 4th Danish Emergency Medicine Conference \\ Roskilde, Denmark. 25-26 November 2011
}

\section{Background}

Early treatment of sepsis has lead to improved outcomes, making early identification of the condition essential. The early warning trigger system TOKS (Tidlig Opsporing af Kritisk Sygdom), including systematically daily recordings of vital signs, was implemented November 1st 2009 throughout Aarhus University Hospital. We hypothesized that the use of TOKS would result in earlier administration of antibiotics for septic patients. The aim of the present study was to compare the degree of appropriately initiated therapy for septic patients admitted to the ICU before and after introduction of TOKS.

\section{Methods}

Our cohort of septic patients was identified through a prospective screening of all patients admitted to the ICU (a 12-bed, tertiary intensive care unit receiving approx. 900 patients/year) from the Emergency Department (ED) and wards. We included all septic patients admitted 3 and 10 months before and after TOKS, respectively. We reviewed patient charts regarding timing of appropriate sepsis therapy (antibiotic administration and blood cultures).

\section{Results}

We identified 78 patients with sepsis admitted to the ICU (46 in the before- and 32 in the after TOKS groups), accounting for $25.3 \%$ resp. $18.1 \%$ of all ICU admissions $(p=0.09)$. A total of 49 patients $(63 \%)$ were admitted directly from the ED. There were no differences regarding age and gender between the two groups. Overall in-hospital mortality was $24.4 \%$.

Appropriate antibiotic treatment was initiated prior to ICU-admission in $37 \%$ of patients before and $66 \%$ after implementation of TOKS $(\mathrm{p}=0.02)$. Blood cultures were obtained prior to ICU-admission in $51 \%$ resp. $67 \%$ of cases $(\mathrm{p}=0.22)$. Compared to current hospital guidelines, antibiotics were delayed in $25 \%$ of cases before TOKS compared to $12 \%$ after $(\mathrm{p}=0.21)$.

\section{Conclusion}

We found that significantly more patients received appropriate antibiotic treatment prior to admission to the ICU after implementation of an early warning trigger system in the ED and wards. With the increasing focus on sepsis treatment, it is not possible to rule out a time effect, but our observational data may indicate that the use of TOKS leads to earlier administration of antibiotic treatment in patients with sepsis.

\section{Author details}

'Research Center for Emergency Medicine, Aarhus University Hospital, Denmark. Intensive Care Unit, Noerrebrogade, Aarhus University Hospital, Denmark.

Published: 16 April 2012

doi:10.1186/1757-7241-20-S2-O3

Cite this article as: Mackenhauer et al:: Does implementation of an early warning trigger system result in earlier administration of antibiotics for septic patients? Scandinavian Journal of Trauma, Resuscitation and Emergency Medicine 2012 20(Suppl 2):O3.

\footnotetext{
* Correspondence: thejuliemac@gmail.com

'Research Center for Emergency Medicine, Aarhus University Hospital, Denmark

Full list of author information is available at the end of the article
} 